\title{
Testes Adaptativos Computadorizados Aplicados em Avaliações
}

\section{Educacionais}

\author{
Title: Computerized Adaptive Testing Applied in Educational Evaluations
}

\section{Débora Spenassato}

Instituto de Matemática, Estatística e Física

Universidade Federal do Rio Grande (FURG)

Rio Grande - RS, Brasil

debospenassato@gmail.com

\section{Dalton Francisco de Andrade}

Departamento de Informática e Estatística

Universidade Federal de Santa Catarina (UFSC)

Florianópolis - SC, Brasil

dalton.andrade@ufsc.br

\author{
Andréa Cristina Trierweiller \\ Programa de Pós-Graduação em Tecnologias da \\ Informação e Comunicação \\ Universidade Federal de Santa Catarina (UFSC) \\ Araranguá - SC, Brasil \\ andreatri@gmail.com
}

\author{
Antonio Cezar Bornia \\ Departamento de Engenharia de Produção e Sistemas \\ Universidade Federal de Santa Catarina (UFSC) \\ Florianópolis - SC, Brasil \\ cezar.bornia@ufsc.br
}

\begin{abstract}
Resumo Os avanços na área da tecnologia contribuíram para aumentar a eficiência da avaliação do processo ensino-aprendizagem com o uso dos Testes Adaptativos Computadorizados (CAT). Esses testes adaptam a avaliação ao nível de conhecimento do respondente e apresentam inúmeras vantagens sobre os testes aplicados de forma convencional, denominados de papel e lápis - P\&P (acrônimo em inglês - Paper \& Pencil), principalmente para os programas que aplicam testes longos, como é o caso do Exame Nacional do Ensino Médio (Enem). Assim, este artigo visa apresentar as vantagens do uso desses testes e compará-los com os escores obtidos via P\&P para o teste da área de Matemática e suas Tecnologias do Enem 2012, composto por 45 itens. Para tanto, uma simulação com dados reais foi realizada. Os escores via aplicação $P \& P$ foram divididos em intervalos para melhor análise da precisão e definição do comprimento do CAT. A partir dos resultados, definiu-se um comprimento de 33 itens para o CAT, o que gera uma redução de pelo menos 26,6\% no comprimento do teste e, consequentemente, redução no tempo do teste e fadiga dos respondentes. Análises sobre o uso dos itens que compõem o banco de itens, também são apresentadas.
\end{abstract}

Palavras-Chave: Testes Adaptativos Informatizados, Avaliação em Larga Escala, Teste de Matemática, Enem, Teoria da Resposta ao item.

\begin{abstract}
Advances in technology have contributed to increase the efficiency of the evaluation on teaching-learning process with the use of Computerized Adaptive Testing (CAT). These tests adapt the evaluation to the respondent's knowledge level and they present several advantages over the conventional tests, called paper and pencil $(P \& P)$, especially for programs implementing long tests, such as Exame Nacional do Ensino Médio - Enem (National High School Exam). Thus, this article aims to present the advantages of the CAT and compare them with the scores obtained from P\&P tests for the "Mathematics and its Technologies" area from Enem 2012, which consists of 45 items. For this, a simulation was carried out with real data. The P\&P scores were divided into intervals for better analyze the accuracy and define CAT length. We obtained a length of 33 items for CAT, which leads to a reduction of at least $26.6 \%$ in test length and hence reduce the test time and the respondents' fatigue. We also present a analysis of the items used.
\end{abstract}

Keywords: Computerized Adaptive Testing, Evaluation in Large Scale, Mathematics Test, Enem, Item Response Theory. 


\section{Introdução}

“A avaliação da aprendizagem é uma atividade fundamental para qualquer organização educacional. [...] A avaliação no seio da atividade de aprendizagem é uma necessidade, tanto para o professor quanto para o aluno" [1, p. 44]. Sendo assim, esta tarefa, quando feita de forma adequada, fornece, entre outras informações, subsídios para o desenvolvimento de novas políticas educacionais, além de permitir detectar possíveis problemas na aprendizagem do aluno e identificar as dificuldades específicas de cada um [2].

Com o avanço da tecnologia computacional nas últimas décadas, novas formas de avaliação têm ganhado destaque, como é o caso dos Testes Adaptativos Computadorizados (CATs - do termo inglês Computerized Adaptive Testing). Esses testes visam melhorar o processo de avaliação da aprendizagem e trazem muitos benefícios, em relação aos testes aplicados de forma convencional, por exemplo, por meio de papel e lápis/caneta ( $\mathrm{P} \& \mathrm{P}-$ do acrônimo em inglês paper and pencil).

O Teste Adaptativo Computadorizado (CAT) é aplicado de forma adaptativa aos respondentes, via computador, cujos itens são selecionados sequencialmente durante o teste, após cada resposta dada a um item, de acordo com regras predefinidas do algoritmo computacional. Essas regras visam especificar o método para iniciar o teste, método de seleção dos itens, método de estimação do escore e critério para finalização do teste ou de parada.

Para sua implementação, é necessário um banco de itens já desenvolvido e com parâmetros dos itens estimados pela Teoria da Resposta ao Item (TRI) [3]. Assim, quando um item é aplicado e o indivíduo responde corretamente, o próximo item a ser apresentado será mais difícil; caso a resposta seja incorreta, o próximo item será um pouco mais fácil que o anterior, e assim por diante, até atingir o critério de finalização do teste. Desta forma, os itens aplicados acabam sendo mais informativos, melhorando a eficiência do teste $\mathrm{e}$ reduzindo o seu comprimento [4].

Pesquisas nesta área têm focado o desenvolvimento de novos métodos para algoritmos do CAT, comparação dos métodos disponíveis e avaliação da comparabilidade entre diferentes formas de aplicação de testes [3]. Orientações a serem seguidas para obter sucesso no desenvolvimento de um CAT podem ser obtidas em estudos mais recentes [5-8].

Atualmente, há um uso expressivo deste método de avaliação em testes na área da saúde, devido a iniciativa dos Institutos Nacionais de Saúde dos Estados Unidos para desenvolver melhores ferramentas para a avaliação em investigação clínica e tratamento de várias doenças (dimensões física, mental e social) [9].

Este método pode ser utilizado pelas Instituições para seleção e classificação de alunos para graduação e pósgraduação, em testes para a seleção de candidatos de uma empresa, para a avaliação de treinamentos ou certificação. Algumas avaliações em larga escala, já implementadas com sucesso e conhecidas no mundo são: Graduate Record Examination (GRE), Graduate Management Admission Test (GMAT), Armed Services Vocational Aptitude Battery (ASVAB), Test of English as a Foreign Language (TOEFL), European Computer Driving Licence (ECDL), National Assessment of Educational Progress (NAEP).

Visando destacar os benefícios que podem ser obtidos por meio da utilização desse método, principalmente para testes em larga escala e para testes longos, este trabalho tem por objetivo comparar os resultados do teste da área de "Matemática e suas Tecnologias" do Exame Nacional do Ensino Médio (Enem, 2012), obtidos via CAT e P\&P, por meio de simulação com dados reais (denominada post-hoc). Destaca-se que, as simulações são muito importantes para definir o design mais adequado do CAT para cada contexto, antes da aplicação efetiva aos respondentes $[10,11]$.

O Exame Nacional do Ensino Médio (Enem) foi utilizado como exemplo, visto que é uma das avaliações brasileiras mais importantes e, a cada ano, milhões de indivíduos se submetem a este teste, que é oferecido anualmente pelo Ministério da Educação e Instituto Nacional de Estudos e Pesquisas Educacionais Anísio Teixeira (Inep), com o objetivo de avaliar o desempenho de estudantes ao fim do Ensino Médio. Os resultados do Enem podem ser utilizados como mecanismo único, alternativo ou complementar de acesso à Educação Superior pelas Instituições de Ensino, para a obtenção de certificados do Ensino Médio e para o acesso a programas oferecidos pelo Governo Federal [12].

O Enem é composto por questões objetivas de quatro áreas gerais, cada uma composta por 45 itens, e uma redação em língua portuguesa. O teste é aplicado de forma convencional (P\&P) e, por ser um teste extenso, sua aplicação é realizada em dois dias, tornando-se cansativo para os indivíduos que se submetem ao teste, que muitas vezes, também acabam reclamando do curto tempo para responder tantos itens, muitos dos quais apresentam um certo grau de dificuldade, que não condiz com o grau de conhecimento do respondente. Nesses casos, os testes adaptativos se tornam uma alternativa para tentar resolver este problema.

Neste contexto, este estudo vem contribuir para 
fomentar a pesquisa nesta área, no Brasil e incentivar a utilização desse método de avaliação, nos mais diversos contextos em que se objetiva mensurar um traço latente, o qual faz uso de aspectos computacionais para se obter resultados mais precisos. Além disso, a utilização de dados de uma situação real, mostra os verdadeiros benefícios que podem ser obtidos com sua implementação, estendendo-se para a avaliação somativa ou formativa, tanto no ensino presencial quanto à distância.

A seguir, apresentam-se os aspectos fundamentais de um CAT, uma revisão sobre o cálculo do escore no Enem e TRI, o método utilizado no estudo, os resultados obtidos e as considerações finais.

\section{Teste Adaptativo Computadorizado}

Os CATs são compostos, basicamente, por cinco componentes: (1) um banco de itens com parâmetros estimados pela TRI, (2) método para iniciar o teste, (3) método de seleção dos itens, (4) método de estimação do escore e (5) critério para finalização do teste. Diversas restrições podem ser impostas no algoritmo, conforme a necessidade de cada teste, uma vez que, dependendo da aplicação, maior segurança e sigilo dos itens serão exigidos. Comumente, restrições de balanceamento de conteúdo e controle da exposição do item são implementadas. Para cada um destes componentes, vários métodos estão disponíveis [13].

O controle da taxa de exposição dos itens é um método utilizado para aumentar a segurança do teste quando eles são sigilosos [14], bem como aumentar o uso dos itens do BI $[15,16,17]$. A taxa de exposição é dada pelo número de indivíduos que responderam o item dividido pelo número total de respondentes. A segurança do BI, também pode ser verificada por meio da análise da sobreposição de testes, que se refere ao número de itens comuns entre os respondentes. Em princípio, quanto mais baixa a taxa de exposição, menor é a quantidade de sobreposição dos itens nos testes [18].

A restrição de balanceamento de conteúdo visa garantir que itens de todos os conteúdos sejam aplicados aos respondentes. Esta estratégia busca maximizar a informação dentro dos domínios para selecionar o próximo item a ser aplicado, controlando o número de itens selecionado, de cada conteúdo [19].

Destaca-se que, a escolha do método mais adequado para cada componente de um CAT irá depender do contexto do teste, do modelo da TRI utilizado e das restrições que precisam ser incluídas [20]. Portanto, torna-se fundamental, nesta fase inicial de elaboração do CAT, estudos de simulação para ajudar a avaliar o desempenho e definir o design do CAT, antes da aplicação do mesmo aos respondentes [10,11]. Por este motivo, simulações com dados reais são efetuadas para testar um CAT [21,22].

Em CAT, o algoritmo computacional, previamente especificado, seleciona os itens de um Banco de Itens (BI) e aplica ao respondente. A administração de um CAT inicia com a aplicação de um ou mais itens, cuja dificuldade inicial é estabelecida e o escore provisório é estimado. Caso o critério de parada não seja satisfeito, um novo item é selecionado e este ciclo se repete [23,24], conforme Figura 1.

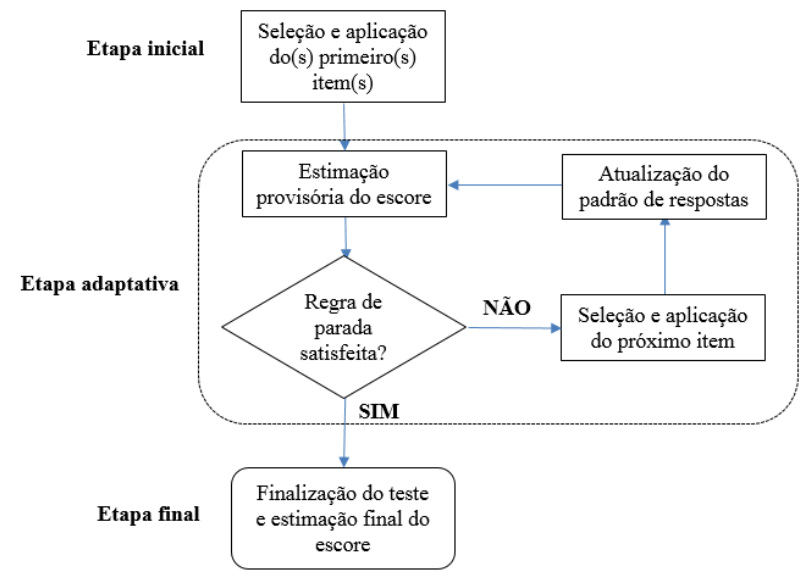

Figura 1: Ideia do algoritmo básico de um CAT. Fonte: Adaptado de Magis e Barrada [25].

O resultado da avaliação é fornecido imediatamente após a finalização, juntamente com relatório detalhando as habilidades e competências apresentadas pelo respondente, benéfico para os professores e alunos, que devido ao uso da TRI, possibilita-se uma interpretação mais rica do escore do respondente, uma vez que coloca itens e respondentes na mesma escala [26-28].

Diferentemente do que ocorre hoje no Enem, em que todos os indivíduos respondem aos mesmos itens, no contexto de um CAT, eles responderão a itens diferentes, mas seus escores serão comparáveis pelo fato dos itens serem selecionados de um banco de itens (BI) calibrados na mesma escala [4,19]. A calibração se refere ao processo de estimação dos parâmetros dos itens pela TRI.

A Figura 2 apresenta uma ilustração da aplicação do CAT a um respondente com escore verdadeiro igual a zero $(\theta=0)$, o qual passou a ser estimado após a aplicação do primeiro item. $\mathrm{O}$ critério de finalização do teste foi fixado em 45 itens; mesmo comprimento do teste de Matemática no Enem. Tem-se o padrão de respostas, em que "zero" significa o erro do item e "um" significa o acerto; a linha vermelha representa a estimativa do escore a cada item aplicado. A linha cinza representa o nível de 
dificuldade do item. A linha tracejada representa a estimativa final do escore.

Observa-se, na Figura 2, que a estimativa do escore está muito próximo do verdadeiro escore; isto é, 0,014 com erro padrão (EP) igual a 0,258 . No entanto, com apenas 23 itens, uma redução de quase $50 \%$ no comprimento do teste, o escore estimado é 0,002 e $\mathrm{EP}=0,343$. Ou seja, muito próximo do verdadeiro.

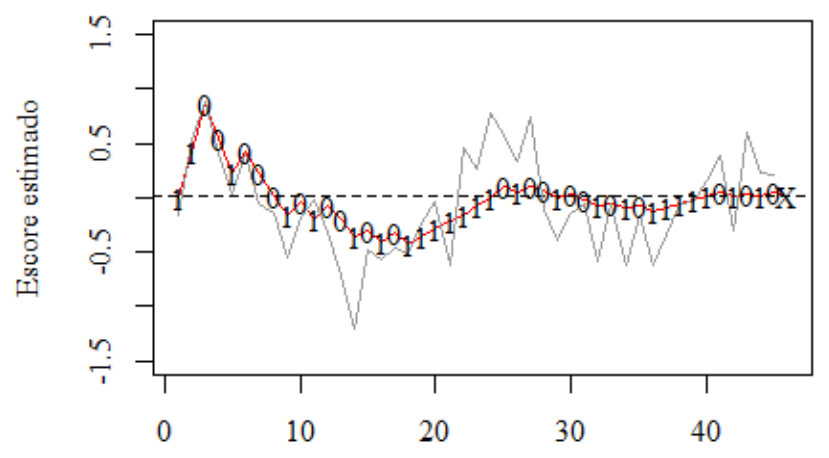

Número de itens

Figura 2: Ilustração da aplicação do CAT a um respondente.

Esta forma de avaliação também possibilita inovações no modo de apresentação dos itens, como anexar várias multimídias (gráficos, áudio ou vídeo), tanto no enunciado quanto nas opções de resposta [29-32]; redução de custos com materiais para o desenvolvimento de testes, armazenamento e correção [5,29]; e benefícios como flexibilidade, baixo custo e facilidade de uso e aplicação, quando os testes podem ser realizados em dispositivos móveis [33].

Por outro lado, em CATs, geralmente o respondente fica impossibilitado de revisar itens ou "pular" e depois retornar para responder, pois estas ações poderiam alterar toda a cadeia de itens aplicados [29,10,34]. Além disso, para aplicação de um CAT, é preciso ter um grande BI e que os respondentes tenham um mínimo de conhecimento em informática $[4,20]$. Em testes de alto impacto como Enem, não seria viável disponibilizar um gabarito ou o teste, visto que cada respondente recebe um teste diferente e, desta forma, todo o BI terá que ser divulgado.

Atualmente, os custos para desenvolvimento e manutenção de um CAT ainda são bastante elevados [16], necessitando do acompanhamento de um grupo multidisciplinar de profissionais para desempenhar estas tarefas.

\section{Teoria da Resposta ao Item e o Escore do Enem}

O Enem é constituído por quatro áreas de conhecimento do Ensino Médio e respectivos componentes curriculares, conforme mostra o Quadro 1. Os itens de múltipla escolha são corrigidos como correto ou incorreto (dicotomizados) e, desde 2009, os parâmetros dos itens e a proficiência/conhecimento dos respondentes, representado pelo escore, são estimados com base no Modelo Logístico Unidimensional de Três Parâmetros (3PL) da TRI, estabelecendo-se a escala com média igual a 500 e desvio-padrão $100 \quad(500,100)$, permitindo comparação do desempenho ao longo do tempo [35].

\begin{tabular}{|l|l|}
\hline $\begin{array}{l}\text { Áreas de } \\
\text { Conhecimento }\end{array}$ & Componentes Curriculares \\
\hline $\begin{array}{l}\text { Ciências Humanas e } \\
\text { suas Tecnologias }\end{array}$ & $\begin{array}{l}\text { História, Geografia, } \\
\text { Filosofia e Sociologia }\end{array}$ \\
\hline $\begin{array}{l}\text { Ciências da } \\
\text { Natureza e suas } \\
\text { Tecnologias }\end{array}$ & Química, Física e Biologia \\
\hline $\begin{array}{l}\text { Linguagens, Códigos } \\
\text { e suas Tecnologias } \\
\text { e Redação }\end{array}$ & $\begin{array}{l}\text { Língua Portuguesa, } \\
\text { Estrangeira (Inglês ou } \\
\text { Física e Tecnologias da } \\
\text { Informação e Comunicação }\end{array}$ \\
\hline $\begin{array}{l}\text { Matemática e suas } \\
\text { Tecnologias }\end{array}$ & Matemática \\
\hline
\end{tabular}

Quadro 1: Áreas de conhecimento e os componentes curriculares avaliadas pelo Enem. Fonte: [12]

A TRI modela a probabilidade de um indivíduo responder corretamente a um item como função dos parâmetros do item e da proficiência do respondente [26,27]. O modelo 3PL, desenvolvido por Birnbaum [28], considera para cálculo do escore do respondente o poder de discriminação (parâmetro $a$ ), a dificuldade (parâmetro $b$ ) e a probabilidade de acerto ao acaso (parâmetro $c$ ) de cada item.

A probabilidade $p_{i j}$ de que o indivíduo $j$ responda corretamente ao item $i$ é dada pela função de resposta ao item (FRI)

$p_{i j}=P\left(Y_{i j}=1 \mid \theta_{j}\right)=c_{i}+\left(1-c_{i}\right) \frac{1}{\left.1+e^{-a_{i}\left(\theta_{j}-b_{i}\right.}\right)}$

onde $i=1, \ldots, I ; j=1, \ldots, n$ e $\theta_{j}$ está associado à proficiência do indivíduo $j$, representado pelo escore do teste. A representação gráfica da FRI é também chamada de curva característica do item $(\mathrm{CCI})$ e determina que, quanto maior a proficiência, maior é a probabilidade de acertar o item. O parâmetro $b_{i}$ é medido na mesma escala da proficiência; $a_{i}>0$ é proporcional à inclinação da CCI no ponto $b_{i}$, e quanto maior o valor deste parâmetro, 
maior será o poder do item em discriminar os indivíduos; $c_{i}$ representa a probabilidade de indivíduos com baixa proficiência responderem corretamente o item $i$, $c_{i} \in[0,1]$.

O método utilizado para o cálculo das proficiências é denominado Esperança a Posteriori (EAP) [36]. Ao final, o respondente recebe cinco escores, um para cada área do teste e o escore da redação, ficando sob responsabilidade da Instituição de Ensino, impor as regras para uso de ingresso. Ou seja, atribui-se pesos distintos, conforme áreas de interesse do curso que está sendo pleiteado ou calcula-se uma média aritmética simples [35].

Esta metodologia de avaliação, utilizada pelo Enem e que é utilizada em CAT, possui vantagens em relação aos procedimentos e modelos clássicos (Teoria Clássica de Testes - TCT) [37,38], que ainda são muito adotados na área educacional, psicológica, da saúde, dentre outras.

A TRI permite a comparação dos escores dos respondentes, mesmo quando diferentes subconjuntos de itens são utilizados, por estarem na mesma escala [26,32]. Devido a essas características, os itens podem ser misturados e combinados com facilidade para produzir formas alternativas ou curtas de um teste, partindo de um mesmo banco de itens [39], facilitando a avaliação de mudanças ao longo do tempo. Além disso, como a precisão é definida para cada nível da proficiência em particular, é possível identificar a faixa para qual o item pode discriminar melhor entre os indivíduos e revelar quão bem, diferentes itens discriminam em diferentes níveis. Esta informação pode ser usada para selecionar os itens mais informativos e administrá-los ao respondente em CAT [40].

\section{Método de pesquisa}

Neste trabalho, foram utilizados dados reais disponíveis e um algoritmo computacional para o CAT, já implementado em pacotes do software R [41]. No entanto, a forma de analisar os dados, buscando a melhor regra de parada para ser utilizada no CAT, foi desenvolvida pelos autores. A seguir, detalham-se os procedimentos utilizados no estudo.

\subsection{Obtenção dos dados e banco de itens}

Os dados reais para serem utilizados na simulação do CAT foram obtidos do Enem 2012, disponibilizados no site do Inep (http://portal.inep.gov.br/basica-levantamentos-

microdados). Para este estudo, apenas dados da prova rosa da área de Matemática e suas Tecnologias foram selecionados. Esta área tem como objetivo avaliar conhecimentos numéricos, algébricos, geométricos e de estatística e probabilidade [12].

Como não é possível obter os parâmetros dos itens na escala do Enem, que utiliza como referência o Enem 2009, fez-se uma nova calibração dos 45 itens, a qual foi respondida por 973.881 indivíduos. Isto é possível, pois o Inep divulga o gabarito e as respostas de cada indivíduo, que respondeu a este teste. A estimação dos parâmetros dos itens e dos escores dos respondentes foi feita por meio do software Bilog [42], o qual fixa as seguintes prioris para os parâmetros de discriminação e de acerto casual: $a_{i} \sim \log$-normal $(1 ; 1,649)$ e $c_{i} \sim \operatorname{beta}(5,17)$.

A dimensionalidade do teste foi verificada pela Análise de Componentes Principais com matriz de correlação tetracórica [43] e análise fatorial de informação completa (full-information) [44], utilizando os pacotes Psych [45] e mirt [46], do software R [41]. Essas técnicas são mais adequadas para dados dicotômicos. Em seguida, verificou-se o ajuste do modelo da TRI aos dados.

Após a estimação dos escores com o BI completo (dados da aplicação do Enem via método P\&P para os 45 itens do teste), estes foram separados em dez intervalos (de -1,9 a 3,5 - vide Tabela 2) e observou-se o erro padrão (EP) das estimativas em cada caso. Isto porque diferentes precisões são obtidas conforme o intervalo a que o respondente pertence, ou seja, em áreas mais informativas do teste, a precisão do escore é maior, em regiões menos informativas, onde não há muitos itens disponíveis naquele nível, a precisão é menor.

Para simulação do CAT, uma amostra aleatória de 500 respondentes foi selecionada, de cada intervalo de escore, totalizando 5.000 respondentes (500 respondentes x 10 intervalos). Para possibilitar as comparações dos escores via CAT, desses respondentes com os escores obtidos via $\mathrm{P} \& \mathrm{P}$ e o $\mathrm{BI}$ completo, considerou-se como "escore verdadeiro", o escore estimado pelo software Bilog [42] via P\&P.

\subsection{Simulação do CAT}

Posteriormente, com o BI formado (parâmetros dos 45 itens estimados), com o vetor de respostas dos 5.000 respondentes selecionados para simulação e seus "escores verdadeiros", foi possível simular o CAT, a partir do pacote catR [47] e analisar os resultados.

Como os mesmos itens são aplicados a todos os respondentes no Enem, não foi estabelecido nenhum método de controle de exposição dos itens no CAT, 
nem a restrição de balanceamento de conteúdo, uma vez que o Inep não divulga a que conteúdo cada item pertence. $\mathrm{O}$ método utilizado para estimar o escore provisório e o escore final foi o método EAP com 10 pontos de quadratura, o mesmo utilizado no software Bilog [42]. A seleção do próximo item foi baseada no método de Máxima Informação de Fisher (MFI). Este método busca maximizar as informações obtidas sobre o respondente, a fim de minimizar o erro da estimativa. Desta forma, seleciona o próximo item como sendo aquele que maximiza a função de informação do item [47].

Inicialmente, buscou-se identificar o número de itens, que deveriam ser aplicados em cada intervalo de escores via CAT, de modo que a precisão das estimativas praticamente se estabilizasse. Para isso, utilizou-se como critério de finalização do teste "length = 45", ou seja, todos os 45 itens foram aplicados aos 5.000 respondentes. No entanto, objetivou-se identificar em que momento a diferença entre as precisões estimadas entre o item atual aplicado $\left(\mathrm{EP}_{i, j}\right)$ e o item anterior $\left(\mathrm{EP}_{i-1, j}\right)$ eram inferior a $5 \% \mathrm{e}$ $1 \%$ da precisão estimada do item anterior, conforme exemplo da equação a seguir:

$$
\left|E P_{i, j}-E P_{i-1, j}\right|<\left|0,01 \times E P_{i-1, j}\right|
$$

onde $i$ corresponde ao item, $i=1, \ldots, 45$; e $j$ corresponde ao respondente, $j=1, \ldots, 500$ (para cada intervalo). $\mathrm{EP}_{i, j}$ corresponde ao erro padrão estimado do escore do respondente $j$, após a aplicação de $i$ itens. Esta pequena diferença na precisão é capaz de informar que, mesmo aplicando mais itens, a precisão tende a se manter estável, ou seja, não irá melhorar significativamente. Assim, o teste pode ser encerrado, sem prejuízos a precisão dos escores.

Posteriormente, após a definição do comprimento do CAT, fez-se uma nova simulação considerando como regra de parada este comprimento do teste. Os resultados foram comparados com os "escores verdadeiros", obtidos por meio da aplicação dos 45 itens, utilizando estatísticas como: raiz do erro quadrático médio (RMSE), correlação e viés. Outros índices referentes ao uso dos itens do BI, também são apresentados.

\section{Resultados}

Os resultados são apresentados em três etapas: (1) desenvolvimento do banco de itens - verificação da dimensionalidade do teste e estimação dos parâmetros do modelo na escala $(0,1)$ e (2) identificação do comprimento do CAT com base nos critérios de precisão (1\% e 5\%), e (3) simulação do CAT com comprimento fixo e comparação com os "escores verdadeiros".

\subsection{Desenvolvimento do banco de itens}

A análise de componentes principais com matriz de correlação tetracórica (Figura 3), do teste de Matemática, demonstrou que há um componente dominante. A full-information apresentou um total de $62,4 \%$ de variância explicada por um fator. Estes resultados sugerem que o teste pode ser considerado como unidimensional, ou seja, que há um traço latente dominante responsável pela realização do teste.

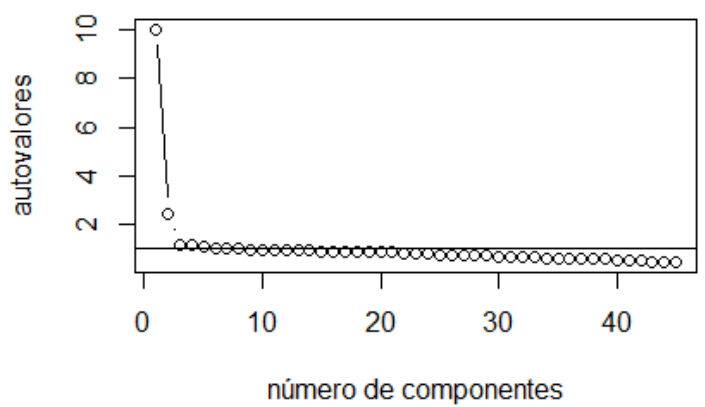

Figura 3: Análise de Componentes Principais

O coeficiente Alfa de Conbrach do teste foi de 0,844 , este coeficiente está relacionado à confiabilidade do teste. A correlação entre os escores em Matemática, divulgado pelo Enem 2012 no site do Inep, e o "escore verdadeiro" estimado pelo software Bilog foi de 0,98 , mostrando uma alta correlação entre os resultados.

Os parâmetros dos itens e respectivos EP de estimação são apresentados na Tabela 1. Nota-se que, os itens 13, 37, 38 e 41 apresentaram altíssimos parâmetros de discriminação $(a>4)$ e baixo EP. De forma geral, a maioria dos itens apresentou bom poder de discriminação, com média de 2,47; apenas os itens 18 e 30 apresentaram $a<0,7$. Os parâmetros de acerto casual variaram de 0,002 a 0,30 .

Em relação aos parâmetros de dificuldade $(b)$, seus valores variaram de $-1,49$ a 2,82. Nota-se que, a maioria dos itens apresentou grau de dificuldade acima de 1 , podendo ser classificados como difíceis. Isso também fica evidente, ao observar a Função de Informação do Teste - FIT (Figura 4), na qual, percebe-se que a mesma está deslocada para a direita da escala. 


\begin{tabular}{|c|c|c|c|c|c|c|c|c|c|c|c|c|c|}
\hline ITEM & & $a$ & & $b$ & & $c$ & ITEM & & $a$ & & $b$ & & $c$ \\
\hline 1 & 0,828 & $(0,009)$ & 1,395 & $(0,009)$ & 0,141 & $(0,003)$ & 24 & 1,258 & $(0,011)$ & 1,721 & $(0,005)$ & 0,163 & $(0,001)$ \\
\hline 2 & 3,511 & $(0,029)$ & 1,568 & $(0,003)$ & 0,269 & $(0,001)$ & 25 & 2,370 & $(0,014)$ & 1,452 & $(0,003)$ & 0,150 & $(0,001)$ \\
\hline 3 & 3,071 & $(0,030)$ & 1,942 & $(0,004)$ & 0,253 & $(0,001)$ & 26 & 1,465 & $(0,008)$ & 0,421 & $(0,005)$ & 0,130 & $(0,002)$ \\
\hline 4 & 3,792 & $(0,036)$ & 1,848 & $(0,003)$ & 0,201 & $(0,000)$ & 27 & 3,025 & $(0,015)$ & 1,003 & $(0,002)$ & 0,177 & $(0,001)$ \\
\hline 5 & 1,688 & $(0,009)$ & 0,367 & $(0,005)$ & 0,219 & $(0,002)$ & 28 & 3,211 & $(0,016)$ & 0,891 & $(0,002)$ & 0,168 & $(0,001)$ \\
\hline 6 & 2,530 & $(0,010)$ & 0,300 & $(0,002)$ & 0,124 & $(0,001)$ & 29 & 2,906 & $(0,027)$ & 1,938 & $(0,004)$ & 0,204 & $(0,001)$ \\
\hline 7 & 2,123 & $(0,009)$ & 0,441 & $(0,003)$ & 0,139 & $(0,001)$ & 30 & 0,500 & $(0,003)$ & 0,796 & $(0,008)$ & 0,003 & $(0,001)$ \\
\hline 8 & 1,862 & $(0,008)$ & $-0,718$ & $(0,006)$ & 0,025 & $(0,004)$ & 31 & 2,446 & $(0,037)$ & 2,472 & $(0,009)$ & 0,188 & $(0,001)$ \\
\hline 9 & 1,329 & $(0,004)$ & $-1,494$ & $(0,004)$ & 0,002 & $(0,001)$ & 32 & 3,297 & $(0,048)$ & 2,420 & $(0,007)$ & 0,126 & $(0,000)$ \\
\hline 10 & 1,708 & $(0,009)$ & $-0,343$ & $(0,007)$ & 0,164 & $(0,003)$ & 33 & 3,314 & $(0,082)$ & 2,757 & $(0,014)$ & 0,129 & $(0,000)$ \\
\hline 11 & 1,519 & $(0,007)$ & $-0,373$ & $(0,006)$ & 0,024 & $(0,003)$ & 34 & 2,520 & $(0,057)$ & 2,823 & $(0,017)$ & 0,168 & $(0,000)$ \\
\hline 12 & 1,622 & $(0,008)$ & 0,045 & $(0,005)$ & 0,106 & $(0,002)$ & 35 & 1,719 & $(0,024)$ & 2,443 & $(0,011)$ & 0,207 & $(0,001)$ \\
\hline 13 & 5,415 & $(0,075)$ & 2,111 & $(0,003)$ & 0,143 & $(0,000)$ & 36 & 2,866 & $(0,038)$ & 2,423 & $(0,007)$ & 0,127 & $(0,000)$ \\
\hline 14 & 1,486 & $(0,011)$ & 1,772 & $(0,005)$ & 0,108 & $(0,001)$ & 37 & 4,152 & $(0,058)$ & 2,227 & $(0,004)$ & 0,176 & $(0,000)$ \\
\hline 15 & 2,166 & $(0,010)$ & $-0,312$ & $(0,004)$ & 0,128 & $(0,002)$ & 38 & 4,544 & $(0,057)$ & 2,006 & $(0,003)$ & 0,208 & $(0,000)$ \\
\hline 16 & 2,157 & $(0,009)$ & 0,012 & $(0,003)$ & 0,092 & $(0,002)$ & 39 & 1,900 & $(0,018)$ & 2,038 & $(0,006)$ & 0,169 & $(0,001)$ \\
\hline 17 & 2,661 & $(0,013)$ & 0,471 & $(0,002)$ & 0,198 & $(0,001)$ & 40 & 2,486 & $(0,024)$ & 1,748 & $(0,004)$ & 0,279 & $(0,001)$ \\
\hline 18 & 0,6481 & $(0,003)$ & 0,366 & $(0,008)$ & $0,004($ & $0,002)$ & 41 & 4,696 & $(0,037)$ & 1,614 & $(0,002)$ & 0,178 & $(0,000)$ \\
\hline 19 & 2,724 & $(0,018)$ & 1,331 & $(0,003)$ & 0,209 & $(0,001)$ & 42 & 3,695 & $(0,021)$ & 1,215 & $(0,002)$ & 0,180 & $(0,001)$ \\
\hline 20 & 2,359 & $(0,012)$ & 1,203 & $(0,002)$ & 0,141 & $(0,001)$ & 43 & 2,044 & $(0,015)$ & 1,325 & $(0,004)$ & 0,304 & $(0,001)$ \\
\hline 21 & 2,931 & $(0,017)$ & 1,182 & $(0,002)$ & 0,187 & $(0,001)$ & 44 & 1,783 & $(0,012)$ & 0,966 & $(0,004)$ & 0,293 & $(0,001)$ \\
\hline 22 & 2,675 & $(0,013)$ & 1,128 & $(0,002)$ & 0,136 & $(0,001)$ & 45 & 1,887 & $(0,010)$ & 0,724 & $(0,003)$ & 0,177 & $(0,001)$ \\
\hline 23 & 2,465 & $(0,012)$ & 1,240 & $(0,002)$ & 0,103 & $(0,001)$ & & & & & & & \\
\hline
\end{tabular}

Tabela 1: Parâmetros dos itens do teste de Matemática do Enem 2012

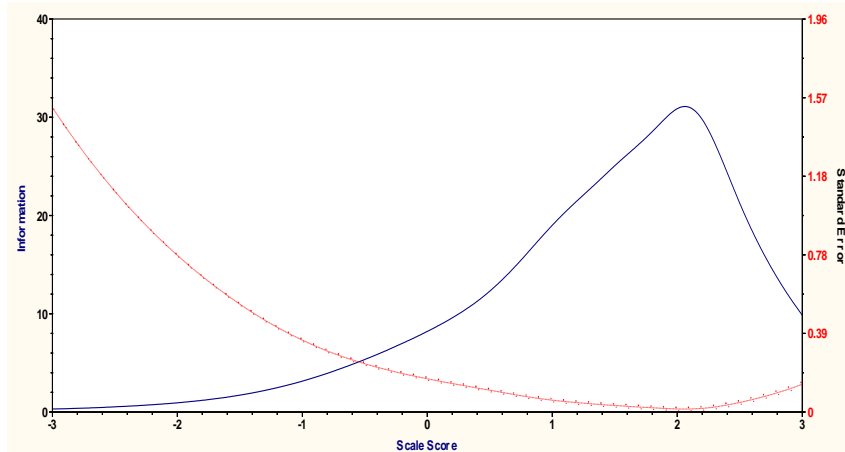

Figura 4: Função de Informação do Teste

A FIT é composta pela soma das funções de informações de cada item que compõem o teste, que informam quanto cada item contribui para a mensuração do escore total [26,37]. Assim, a FIT fornece o intervalo em que o teste é capaz de medir com maior precisão o escore dos respondentes. A linha tracejada representa o EP de medida nos diferentes pontos da escala. Neste caso, o teste é capaz de mensurar com maior precisão o escore dos respondentes acima da média zero.

A FIT também pode ser utilizada para fornecer informações sobre a regra de parada a ser adotada quando o CAT tem comprimento variável, por exemplo, pela definição da regra de parada, com base na precisão do escore. Assim, quando o respondente atingir a precisão preestabelecida para o escore, o teste é encerrado.

Considerando que o EP de medida é diferenciado, dependendo do local da escala em que o respondente se encontra, optou-se por separar os escores em intervalos para melhor análise das estimativas (Tabela 2) e da quantidade de itens necessária em cada caso, na simulação do CAT.

A média do EP dos "escores verdadeiros" foi de 0,34 e mediana de 0,37 para os 45 itens. Assim, com base na Figura 4, pode-se observar que, se fosse estabelecido uma regra de parada com precisão igual a média ou mediana do EP, indivíduos que estão localizados no extremo inferior da escala poderiam nunca atingir este critério, uma vez que, não há itens suficientes no BI para estimar com esta precisão o escore desses indivíduos. Portanto, 
seria necessário acrescentar novos itens neste ponto da escala. Isso também fica claro ao observar o EP nos intervalos de $-1,9$ a -1 , na Tabela 2 .

Conforme destacam Veldkamp e Matteucci [16], em aplicações do CAT no contexto de certificação, nivelamento e classificação, como é o caso do Enem, costumam-se utilizar um número fixo de itens, pois um comprimento variável, nesses casos, pode não ser viável, porque os respondentes podem perceber o teste como injusto, dado que cada respondente poderá receber um comprimento de teste diferente.

\begin{tabular}{|c|c|c|c|c|c|}
\hline & $\begin{array}{c}\text { Intervalo de } \\
\text { proficiência } \\
\text { (escores) }\end{array}$ & $\begin{array}{c}\text { Tamanho da amostra } \\
\text { no intervalo }\end{array}$ & EP mínimo & EP máximo & Média do EP \\
\hline 1 & {$[-1,9$ a 1,5$]$} & 43.636 & 0,47 & 0,69 & 0,58 \\
\hline 2 & ]$-1,5 ;-1]$ & 108.698 & 0,42 & 0,84 & 0,53 \\
\hline 3 & ]$-1 ;-0,5]$ & 175.211 & 0,23 & 0,80 & 0,43 \\
\hline 4 & ]$-0,5 ; 0]$ & 164.705 & 0,18 & 0,83 & 0,37 \\
\hline 5 & ] $0 ; 0,5]$ & 256.803 & 0,04 & 0,66 & 0,24 \\
\hline 6 & ] $0,5 ; 1]$ & 56.211 & 0,22 & 0,72 & 0,37 \\
\hline 7 & ] $1 ; 1,5]$ & 132.248 & 0,00 & 0,45 & 0,15 \\
\hline 8 & ] $1,5 ; 2]$ & 8.588 & 0,35 & 0,45 & 0,41 \\
\hline 9 & ] $2 ; 2,5]$ & 26.469 & 0,01 & 0,41 & 0,12 \\
\hline 10 & ] $2,5 ; 3,5]$ & 1.312 & 0,17 & 0,44 & 0,31 \\
\hline
\end{tabular}

Tabela 2: Estatísticas descritivas relacionadas ao EP dos "escores verdadeiros" em cada intervalo

\subsection{Identificação do comprimento do CAT}

Para simulação do CAT, tem-se que o BI possui 45 itens de Matemática com os parâmetros apresentados na Tabela 1 e uma amostra de 5.000 respondentes. Para iniciar o teste, quatro itens iniciais foram selecionados de uma variação de proficiência inicial definida entre -2 e 2 e aplicados; posteriormente, a proficiência passou a ser estimada a cada item aplicado.

Neste contexto de simulação, todos os itens foram apresentados. Porém, o objetivo era identificar durante este processo, onde (com quantos itens) o EP praticamente se estabilizava. É esperado que, conforme a aplicação de itens avança, o EP tende a reduzir gradualmente e essa desaceleração, significa que os itens que restam no banco são pouco informativos para o nível de proficiência do respondente [48].

Os resultados obtidos em relação ao número de itens, em que se atenda aos critérios já definidos anteriormente, são apresentados na Tabela 3. Observa-se que, no intervalo de proficiências em que há maior precisão dos "escores verdadeiros", o número de itens exigidos para que a diferença entre os EP estimados seja pequena é maior. Além disso, pode-se observar que o número de itens necessários variou entre 11 e 33 para o critério mais cauteloso de $1 \%$.

Para o critério de $1 \%$, o número máximo de itens para o teste de Matemática do Enem 2012 é 33 itens, significando uma redução de pelo menos $26,6 \%$ no comprimento do teste e na economia de itens do BI. Considerando o critério de $5 \%$, tem-se uma variação entre 7 e 23 itens. Ou seja, uma redução de, pelo menos, $48,8 \%$ no comprimento do teste.

\begin{tabular}{c|c|c}
\hline $\begin{array}{c}\text { Intervalo de } \\
\text { proficiência } \\
\text { (escores) }\end{array}$ & $\begin{array}{c}\text { Num. de itens } \\
\text { para critério } \\
\text { de 5\% }\end{array}$ & $\begin{array}{c}\text { Num. de itens } \\
\text { para critério } \\
\text { de 1\% }\end{array}$ \\
\hline$[-1,9 a 1,5]$ & 7 & 11 \\
\hline$]-1,5 ;-1]$ & 8 & 15 \\
\hline$]-1 ;-0,5]$ & 10 & 19 \\
\hline$]-0,5 ; 0]$ & 11 & 24 \\
\hline$] 0 ; 0,5]$ & 19 & 26 \\
\hline$] 0,5 ; 1]$ & 17 & 32 \\
\hline$] 1 ; 1,5]$ & 18 & 32 \\
\hline$] 1,5 ; 2]$ & 23 & 33 \\
\hline$] 2 ; 2,5]$ & 23 & 21 \\
\hline$] 2,5 ; 3,5]$ & 16 & 32 \\
\hline
\end{tabular}

Tabela 3: Número de itens para cada intervalo de escores

\subsection{Simulação do CAT}

Após a identificação do número de itens necessários para o teste, fez-se uma nova simulação, supondo que os 5.000 respondentes foram submetidos ao teste, com critério de parada fixado em 33 itens pelo critério mais cauteloso de $1 \%$ na variação do EP.

Nesta situação, tem-se os seguintes resultados: o RMSE dos escores foi de 0,088; a correlação entre "escores verdadeiros" e estimados via CAT foi de 0,998 ; o viés médio foi igual a 0,00003 , o EP médio das estimativas dos escores com 33 itens foi de 0,351 , estando muito próximo a 0,34 , valor obtido com a aplicação de todo o BI, e o intervalo de precisão foi de 
0,010 a 0,684 . A Figura 5 mostra que a maior diferença entre os "escores verdadeiros" e os escores estimados pelo CAT ocorrem no intervalo onde há maior informação no BI. Ou seja, escores entre 1 e 2,5, visto que, se fossem aplicados mais do que 33 itens nessa região, as estimativas seriam mais próximas dos "escores verdadeiros".

$\mathrm{Na}$ Figura 5, também é possível observar a representação da exposição dos itens versus parâmetro $a$. Nota-se que, nove itens foram expostos a todos os respondentes (itens $8,9,13,14,19,20,23,28$ e 44 ), os quais possuem parâmetros de discriminação acima de 1 . A taxa de sobreposição do teste foi de 0,829 . Esta taxa é considerada alta para testes que exigem níveis elevados de segurança do BI, devendo ser substituído substituída em aplicações futuras. Porém, esta taxa elevada é esperada porque o BI utilizado neste estudo é pequeno (45 itens).
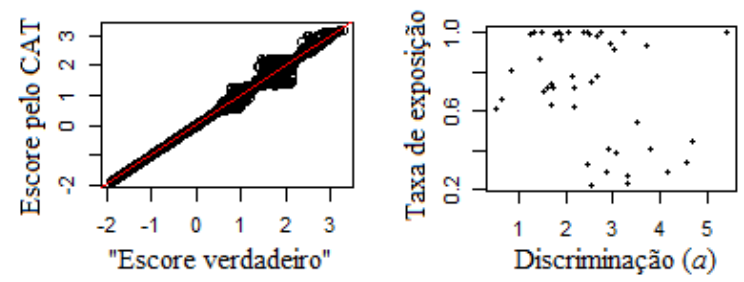

Figura 5: Comparação das estimativas dos escores para os diferentes métodos e taxa de exposição dos itens conforme discriminação dos itens

Em relação ao uso do banco de itens, verificou-se que a taxa mínima de exposição dos itens foi de 0,227 , ou seja, todos os itens do BI foram apresentados pelo menos uma vez aos indivíduos. Isso ocorreu porque os itens possuem bons parâmetros de discriminação e porque o BI é pequeno. Na literatura, a subutilização de alguns itens do BI nos programas de testes é vista com preocupação, uma vez que há gastos com o desenvolvimento desses itens, que acabam sendo pouco ou nunca aplicados, consequentemente, outros itens acabam sendo muito expostos, gerando riscos para testes que exigem alta segurança do BI para não comprometer os resultados da avaliação $[16,19]$.

A Figura 6 representa a simulação de um indivíduo respondendo ao CAT com 33 itens. Observa-se que o escore estimado ficou próximo ao "escore verdadeiro" de 3,207 para este respondente. O valor estimado pelo CAT foi de 3,158 com EP de 0,218, pois ele está no nível em que há vários itens disponíveis. No entanto, nota-se que, com 20 itens o teste já poderia ser encerrado, pois já estaria muito próximo do "escore verdadeiro" deste indivíduo. Assim, este tamanho de teste está próximo ao número de itens esperado para o intervalo de 2,5 a 3,5, para critério de 1\% (conforme Tabela 3), isto é, 21 itens.

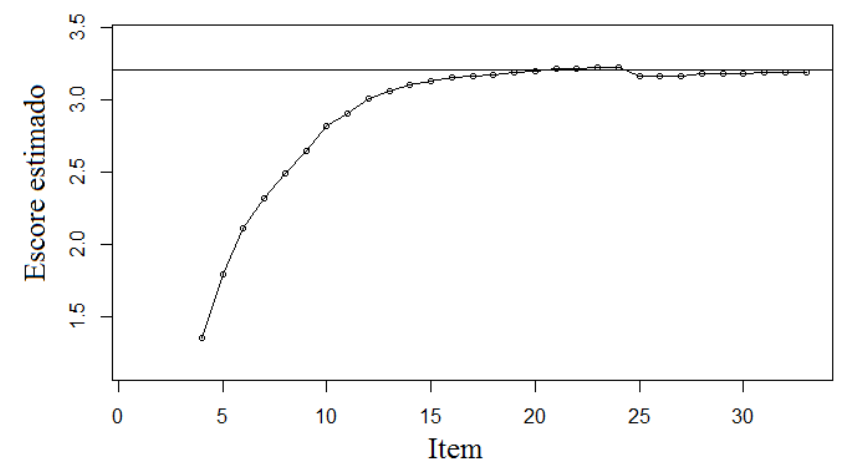

Figura 6: CAT de 33 itens para um respondente do teste

Para os casos em que é possível estabelecer o comprimento variável de um CAT para os respondentes, ou seja, regra de parada com base em uma precisão predefinida ou número máximo de itens aplicados para quem não atinge a precisão, as vantagens apresentadas pelo uso do CAT são ainda maiores. No exemplo da Figura 6, o teste teria uma redução de mais de $50 \%$ do teste original, aplicado pelo Enem.

\section{Considerações Finais}

O CAT tem sido considerado como uma abordagem promissora para aumentar a eficiência na avaliação assistida por computador, proporcionando vantagens para os estudantes e professores, que podem direcionar seu ensino para as dificuldades dos estudantes e elaborar suas ações de forma eficaz. Para os desenvolvedores de testes, pode-se reduzir custos com correção, impressão dos testes, distribuição, etc. Esses testes podem ser aplicados em diversas áreas do conhecimento, tanto para âmbito de avaliação formativa quanto somativa.

A partir deste estudo, foi possível observar que o teste de Matemática do Enem 2012, se aplicado na forma adaptativa (CAT), poderia ser reduzido em, pelo menos, $26,6 \%$ sem perda significativa de precisão, se comparado ao teste completo com 45 itens. Essa redução no comprimento do teste pode diminuir a fadiga dos respondentes, que poderia comprometer os resultados do teste, além de não desestimular o estudante, uma vez que ele não precisará responder a itens muito fáceis ou muito difíceis, para seu nível de conhecimento.

Nesta situação avaliada, não é adequado definir uma regra de finalização do teste baseada apenas em uma precisão comum a todos os respondentes, uma vez que a precisão apresenta valores bem variados, dependendo do nível da escala em que o respondente se encontra e, principalmente, porque a escala possui maior precisão para mensurar o conhecimento dos indivíduos que possuem proficiência acima da média. Portanto, se o EP 
fixado para finalizar o teste for muito pequeno, alguns respondentes não irão atingir esta regra.

Este estudo utilizou apenas dados da área de Matemática. No entanto, no contexto geral do Enem, poderiam ser aplicados CATs para cada área do conhecimento em separado, como ocorre atualmente via método convencional de aplicação dos testes. Assim, o escore final pode ser criado pela combinação de um número de mini-CATs [49].

No Brasil, ainda não há aplicações em larga escala de CATs, mas a tendência é que este cenário mude, visto que é um método que traz muitos benefícios em relação aos testes convencionais e há uma crescente divulgação de pesquisas, que vêm utilizando este modo de avaliação. Por isso, estudos com esta temática são importantes para definição do design do CAT que se pretende aplicar, visando todos os benefícios que este teste pode trazer aos respondentes, avaliadores, desenvolvedores de itens, dentre outros. No entanto, é importante destacar que esta é uma tarefa que envolve planejamento, implementação e manutenção contínua.

Para estudos futuros, sugere-se comparar diferentes métodos de seleção dos itens e inserção de restrição para a taxa exposição dos itens e assim, verificar o impacto nas estimativas dos escores.

\section{Referências}

[1] L. L. Lazzarotto, A. P. Oliveira, J. L. Braga, F. J. V. Passos. A educação em ambientes virtuais: proposição de recursos computacionais para aumentar a eficiência do processo ensinoaprendizado. Revista Brasileira de Informática na Educação, 19(2): 42-55, 2011.

[2] L. A. M. Zaina, W. V. Ruggiero, G. Bressan. Metodologia para Acompanhamento da Aprendizagem através da Web. Revista Brasileira de Informática na Educação, 12(1): 1-13, 2004.

[3] D. Spenassato, A. C. Bornia, R. Tezza. Computerized Adaptive Testing: A Review of Research and Technical Characteristics. IEEE Latin America Transactions, 13(12): 3890-3898, 2015.

[4] J. A. Kopec, M. Badii, M. McKenna, V. D. Lima, E. C. Sayre, M. Dvorak. Computerized adaptive testing in back pain - Validation of the CAT-5DQOL. Spine, 33(12): 1384-1390, 2008.

[5] F. J. Moreira Junior. Sistemática para a implantação de testes adaptativos informatizados baseados na teoria da resposta ao item. Tese de doutorado, Universidade Federal de Santa Catarina, 2011.

[6] J. S. Lai, D. Cella, S. Choi, D. U. Junghaenel, C. Christodoulou, R. Gershon, A. Stone. How Item Banks and Their Application Can Influence Measurement Practice in Rehabilitation Medicine: A PROMIS Fatigue Item Bank Example. Archives of Physical Medicine and Rehabilitation, 92(10): S20-S27, 2011.

[7] T. A. Davey. Guide to Computer Adaptive Testing (CAT) Systems. Washington, DC: Council of Chief State School Officers, 2011.

[8] M. D. Anatchkova, M. Rose, J. E. Ware JR., J. B. Bjorner. Development of an item bank and computer adaptive test for role functioning. Quality of Life Research, 21(9): 1625-1637, 2012.

[9] P. A. Pilkonis, et al. Validation of the depression item bank from the Patient-Reported Outcomes Measurement Information System (PROMIS ${ }^{\circledR}$ ) in a three-month observational study. Journal of Psychiatric Research, 56:112-119, 2014.

[10] T. Wang, M. J. Kolen. Evaluating Comparability in Computerized Adaptive Testing: Issues, Criteria and an Example. Journal of Educational Measurement, 38(1): 19-49, 2001.

[11]H. H. Chang, Z. Ying. Nonlinear sequential designs for logistic item response theory models with applications to computerized adaptive tests. Annals of Statistics, 37(3): 1466-1488, 2009.

[12] Brasil. Ministério da Educação - Inep. Edital Enem $2014 . \quad \mathrm{http} / /$ download.inep.gov.br/ educacao_basica/enem/edital/2014/edital_enem_20 14.pdf. Mar. 2015

[13]E. Georgiadou, E. Triantafillou, A. A. Economides. A Review of Item Exposure Control Strategies for Computerized Adaptive Testing Developed from 1983 to 2005. The Journal of Technology, Learning, and Assessment, 5(8): 1-38, 2007.

[14] M. Finkelman, M. L. Nering, L. A. Roussos. Conditional Exposure Control Method for Multidimensional Adaptive Testing. Journal of Educational Measurement, 46(1): 84-103, 2009.

[15] A. J. Leroux, M. Lopez, I. Hembry, B. G. Dodd. A Comparison of Exposure Control Procedures in CATs Using the 3PL Model. Educational and Psychological Measurement, 73(5): 857-874, 2013.

[16]B. P. Veldkamp, M. Matteucci. Bayesian computerized adaptive testing. Ensaio, 21(78): 57- 
$82,2013$.

[17] J. R. Barrada, F. J. Abad, B. Veldkamp. Comparison of methods for controlling maximum exposure rates in computerized adaptive testing. Psicothema, 21(2):313-20, 2009.

[18] M. L. Stocking. Three Practical Issues for Modern Adaptive Testing Item Pools. Princeton, NJ: Institution Educational Testing Service, Report No ETS-RR-94-5, 1994.

[19]R. M. Luecht, A. De Champlain, R. J. Nungester. Maintaining Content Validity in Computerized Adaptive Testing. Advances in Health Sciences Education, 3(1): 29-41, 1998.

[20]T. Wang, W. P. Vispoel. Properties of ability estimation methods in computerized adaptive testing. Journal of Educational Measurement, 35(2): 109-135, 1998.

[21] J. B. Bjorner, C. H. Chang, D. Thissen, B. B. Reeve. Developing tailored instruments: item banking and computerized adaptive assessment. Quality of Life Research, 16: 95-108, 2007.

[22] K. F. Cook, S. W. Choi, P. K. Crane, R. A. Deyo, K. L. Johnson, D. Amtmann. Letting the CAT out of the bag: Comparing computer adaptive tests and an 11-item short form of the roland-morris disability questionnaire. Spine, 33(12): 1378-1383, 2008.

[23]H. Ozyurt, O. Ozyurt, A. Baki, B. Guven. An Application of Individualized Assessment in Educational Hypermedia: Design of Computerized Adaptive Testing System and its Integration Into UZWEBMAT. Procedia - Social and Behavioral Sciences, 46: 3191-3196, 2012.

[24]H. Wainer. CATs: whit her and whence. Psicologica, 21: 121-133, 2000.

[25]D. Magis, J. R. Barrada. Open-source CAT software: R packages and Concerto. SOQOL-NL, Utrecht, 2014. Disponível em: http://www.isoqol.nl/sites/default/files/Symposia\% 20bestanden/David\%20Magis\%20Open\%20source \%20CAT\%20software.pdf. Acesso em: ago. 2015.

[26]D. F. Andrade, H. R. Tavares, R da C. Valle. Teoria da Resposta ao Item: Conceitos e Aplicações. São Paulo: Associação Brasileira de Estatística, 2000.

[27] S. L. V. Bortolotti, R. Tezza, D.F. Andrade, A. C. Bornia, A. F. Sousa Júnior. Relevance and advantages of using the item response theory. Quality \& Quantity, 47: 2341-2360, 2013.
[28]A. Birnbaum. Some latent trait models and their use in inferring an examinee's ability. In F. M. Lord, M. R. Novick, (eds.) Statistical theories of mental test scores. Reading, MA: Addison-Wesley, páginas 397-479, 1968.

[29]H. Wainer. CATs: whit her and whence. Psicologica, 21: 121-133, 2000.

[30] Y-M. Huang, Y-T. Lin, S-C Cheng. An adaptive testing system for supporting versatile educational assessment. Computers and Education, 52(1): 5367, 2009.

[31]C. G. Parshall, J. C. Harmes, T. Davey, P. J. Pashley. Innovative Items for Computerized Testing. In W. J. van der Linden, C. A. W. Glas (eds.), Elements of Adaptive Testing, Statistics for Social and Behavioral Sciences, páginas 215-230, 2010.

[32] M. C. Edwards. An Introduction to Item Response Theory using the Need for Cognition Scale. Social and Personality Psychology Compass, 3(4): 507529, 2009.

[33] M. D. Anatchkova, M. Rose, J. E. Ware JR., J. B. Bjorner. Development of an item bank and computer adaptive test for role functioning. Quality of Life Research, 21(9): 1625-1637, 2012.

[34] S. L. Wise. The Utility of Adaptive Testing in Addressing the Problem of Unmotivated Examinees. Journal of Computerized Adaptive Testing, 2(1): 1-18, 2014.

[35] Brasil. Ministério da Educação - Inep. Guia do participante - entenda a sua nota no Enem, BrasíliaDF, 2012. http://portal.inep.gov.br/web/ enem/enem. Mar. 2015.

[36]R. D. Bock, R. J. Mislevy. Adaptive EAP estimation of ability in a microcomputer environment. Applied Psychological Measurement, 6: 431-444, 1982.

[37]R. K. Hambleton, H. Swaminathan, J. Rogers. Fundamentals of Item Response Theory. Measurement Methods for the Social Science. Newbury Park, CA: SAGE Publications, 1991.

[38]R. J. De Ayala. The theory and practice of item response theory. New York, NY: Guilford, 2009.

[39]D. Thissen, B. B. Reeve, J. B. Bjorner, C. H. CHANG. Methodological issues for building item banks and computerized adaptive scales. Quality of Life Research, 16: 109-119, 2007.

[40]H. Fliege, J. Becker, O. B. Walter, M. Rose, J. B. Bjorner, B. F. Klapp. Evaluation of a computer- 
adaptive test for the assessment of depression (DCAT) in clinical application. International Journal of Methods in Psychiatric Research, 18(1): 23-36, 2009.

[41] R Core Team. R: A language and environment for statistical computing. R Foundation for Statistical Computing, Vienna, Austria, 2014. http://www.Rproject.org/.

[42] M. Zimowski, E. Muraki, R. Mislevy, R. D. Bock. Software BILOG-MG V3.0. Scientific Software International, Inc. 2003.

[43]R. J. Mislevy. Recent developments in the factor analysis of categorical variables. Journal of Educational and Behavioral Statistics, 11(1): 3-31, 1986.

[44]R. D. Bock, R. Gibbons, E. Muraki. FullInformation item factor analysis. Applied Psychological Measurement, 12(3): 261-280, 1988.

[45]W. Revelle. Procedures for Psychological, Psychometric, and Personality Research. Package: psych. Version: 1.4.8.11, 2014.

[46]R. P. Chalmers. mirt: A Multidimensional Item Response Theory Package for the R Environment. Journal of Statistical Software, 48(6): 1-29, 2012.

[47]D. Magis, G. Raiche. Random Generation of Response Patterns under Computerized Adaptive Testing with the R Package catR. Journal of Statistical Software, 48(8): 1-31, 2012.

[48] C. H. S. S. Nunes, D. Spenassato, C. M. Oliveira, A. C. Bornia, R. Primi. . Testes Adaptativos Computadorizados - CAT. In M. C. R. da Silva, D. Batholomeu, C. M. M. Vendramini, J. M. Montiel (eds.), Aplicações de métodos estatísticos avançados à avaliação psicológica e educacional. 1ed. São Paulo: Vetor, páginas 37-76, 2015.

[49] D. L. Schnipke, B. F. Green. A comparison of item selection routines in linear and adaptive tests. Journal of Educational Measurement, 32(3): 227242, 1995. 